\title{
Severity of Chest Pain among Acute Myocardial Infarction Patients with Diagonal Branch Vessel Disease: A Pilot Study
}

\author{
Muhammad Nasir Rahman ${ }^{1}$, Azmina Artani ${ }^{2}$, Farhala Baloch ${ }^{1}$, Bilal Hussain ${ }^{1}$ \\ 1. Cardiology, The Aga Khan University, Karachi, PAK 2. Epidemiology, The Aga Khan University, Karachi, PAK
}

Corresponding author: Muhammad Nasir Rahman, nasir.rahman@aku.edu

\begin{abstract}
Introduction: Acute myocardial infarction (AMI) patients present with variable clinical manifestations such as shortness of breath, nausea, etc. among which chest pain is the most common. Previous studies have reported that the clinical presentation of AMI patients with branch vessel disease is indistinguishable from epicardial coronary vessel disease. However, our experience suggests patients with branch vessel disease experience severe chest pain, especially those with a diagonal branch. Therefore, we aim to study the association of chest pain severity with isolated diagonal branch vessel disease as a culprit vessel in AMI patients.
\end{abstract}

Methods: It is a retrospective case-control design, where 10 cases and 40 historic controls were recruited in the study. Cases were patients with isolated diagonal branch disease, whereas controls were patients with epicardial vessel disease in AMI. We reviewed Coronary Angiograms of adult patients who presented with acute myocardial infarction and had undergone coronary angiography at Aga Khan University Hospital, Karachi (AKUH). Information on pain scores was measured using the Numeric Pain Rating Scale (NRS) before administration of analgesics. Other relevant variables were also recorded on a pre-structured questionnaire.

Results: The mean age of all the participants in the study was $60 \pm 11.0$ years, with $16 \%$ of the patients being women. Among all AMI patients, the intensity of chest pain in patients with isolated diagonal branch vessel disease was 2.6 units higher as compared to those with other epicardial coronary vessel diseases ( $\mathrm{p}$-value: $<0.001 ; 95 \%$ CI: 1.67 - 3.46).

Conclusion: This preliminary study indicates severe chest pain can be a differentiating symptom in AMI patients with diagonal branch disease. It emphasizes clinicians to look for a possibility of a diagonal branch as a culprit vessel in AMI for better judgment, as it is often overlooked. future studies may be conducted at multiple centers for larger sample size and better generalizability.

Received 07/09/2019

Review began 08/18/2019

Review ended 08/26/2019

Published 08/29/2019

๑) Copyright 2019

Rahman et al. This is an open access article distributed under the terms of the Creative Commons Attribution License CC-BY 3.0., which permits unrestricted use, distribution, and reproduction in any medium, provided the original author and source are credited.
Categories: Cardiology

Keywords: chest pain, diagonal branch vessel disease, acute myocardial infarction

\section{Introduction}

Acute myocardial infarction (AMI) is one of the leading causes of death worldwide [1]. It results from plaque rupture in coronary arteries. Patients with acute myocardial infarction may experience a range of symptoms such as shortness of breath, sweating, and nausea. Chest pain is considered as one of the classical signs of AMI [2].

Chest pain characteristics such as intensity, onset, relieving and aggravating factors may differ from patient to patient [3]. Many researchers across the globe have contributed their knowledge to this phenomenon and have tried to explain the mechanism behind cardiac pain perception [4]. Despite available literature, there is a limited understanding of exact pain pathways and precipitating factors to pain, such as the intensity of pain with respect to the region of myocardium being affected. Pain intensity may also differ across different regions of the heart because of the variable amount of blood supply [5-6].

In our practice, we often noticed patients with occlusion of a diagonal branch [branch arising from the left anterior descending artery (LAD)] to present with more severe chest pain as compared to patients with occlusion of other major epicardial vessels [left circumflex (LCX) and right coronary artery (RCA)] as infarctrelated artery. In these patients, without suggestive findings of occlusion in epicardial vessels, there is a high likelihood of missing the involvement of a diagonal branch as a culprit vessel. In such circumstances, it may lead to a diagnostic dilemma if significant attention is not given while interpreting coronary angiograms. Therefore, in this pilot study, we aimed to study the association of intensity of pain with isolated diagonal branch vessel disease as compared to epicardial vessel disease in AMI patients. 


\section{Materials And Methods \\ Study design and population}

The study incorporated a case-control design and was conducted at the Aga Khan University Hospital, Karachi (AKUH). Data was extracted for patients who presented with AMI to the emergency department at AKUH from the period of June 2014 to June 2016. Participants who were $\geqslant 18$ years and had complete medical records were included in the study. We excluded patients who were on mechanical ventilation, had received analgesics prior coming to the emergency department from health care facilities outside AKUH, had collateral circulation to the obstructed diagonal branch or epicardial vessel, and those with known symptomatic neuropathy. Patients who were known opioid addicts were also excluded as they may have altered pain thresholds because of opioid use.

We defined cases as patients who had isolated diagonal branch, without the involvement of LAD or any other major vessels (such as LCX and RCA). Controls were defined as patients whose coronary angiogram revealed other epicardial coronary vessels (LAD (without the involvement of the diagonal branch), LCX, and RCA).

\section{Data collection}

We began with the identification of cases during the period of June 2015 to June 2016 by reviewing coronary angiograms of AMI patients brought to the cardiac catheterization lab. Upon ascertainment of eligibility criteria, cases and controls were enrolled in the study. As we identified each case, we randomly selected four historic controls upon reviewing the coronary angiograms of previous AMI patients during the study period.

\section{Ascertaining outcome: pain assessment}

Our hospital utilizes the Numeric Pain Rating Scale (NRS) as the most common tool for quantifying pain perception among adults with no cognitive or communication difficulties [7]. The NRS pain scale requires patients to assign a numeric value to the pain from a scale of 0 - 10 where 0 represents no pain and 10 represents maximum pain that they must be having [8].

As a standard of care, upon initial assessment of vital signs, a trained doctor or nurse assesses the pain of all AMI patients using the NRS scale. Electrocardiogram (ECG) of these patients is then performed in order to reach an affirmative diagnosis of AMI. Pain medications are administered to the patients before shifting them to the cardiac catheterization lab for the intervention. These initial assessments are documented in the patient's progress notes, which become part of the hospital record upon the patient's discharge. For this study, the maximum pain score of each patient before the administration of analgesics prior to the procedure was recorded. Information on the history of co-morbid conditions and interpretation of ECG at the time of arrival was also noted.

Ethical approval was sought from the Ethics Review Committee, AKU (4358-Car-ERC-16) which granted an exemption for ethical approval and permission to review the hospital records for this study.

\section{Sample size and statistical analysis plan}

The prevalence of diagonal branch vessel disease as culprit vessel in AMI is very low in the general population, i.e., $0.5 \%-1 \%[9-10]$. Therefore, a total sample of 50 patients was demised sufficient employing a case-control design (cases and controls to be selected in a ratio of 1:4). We assumed a $35 \%$ of severe pain exposure among controls [11-12], to achieve a minimum power of $80 \%$ and $95 \%$ confidence level. The sample size was calculated using OpenEpi Software.

For continuous variables, we report means and standard deviations for normally distributed variables, whereas, for non-normally distributed variables, we report median and interquartile range. For categorical variables, we determined frequencies and proportions. To examine the difference in baseline characteristics of quantitative variables, t-test for an independent sample was applied. Chi-square or Fisher Exact test were applied for categorical variables, as appropriate. A multiple linear regression was performed to determine the association of pain intensity between cases and controls keeping pain score as an outcome, while controlling for other factors and comorbidity profile of participants. Confounding and interaction were also assessed for biologically plausible variables. Data were analyzed using STATA version 12 . A p-value of $\leqslant 0.05$ was deemed significant.

\section{Results}

A total of 50 participants (10 cases and 40 controls) were included in the study upon review of the records. Among controls, $55 \%$ of the patients had LAD, $25 \%$ had left circumflex and $20 \%$ had RCA as culprit vessel in AMI. As the continuous variables in the data followed a symmetrical distribution, we report means \pm standard deviation here. Cases and controls had a similar distribution of characteristics with respect to age, gender, co-morbid conditions (diabetes, dyslipidemia and family history of coronary artery disease (CAD)) and smoking status. However, more of the patients had been hypertensive in the control group (Table 1 ). The mean pain score among the cases was $9 \pm 1.1$ as compared to that of $6 \pm 1.1$ among controls and was 


\section{Cureus}

statistically significant (t-test p-value $<0.001)$.

\begin{tabular}{|c|c|c|c|}
\hline Variables & Cases $(n=10)$ & Controls $(n=40)$ & P-value for Difference \\
\hline Age in years (Mean, \pm SD) & $60.1(10.7)$ & $59.8(11.7)$ & 0.92 \\
\hline Female gender (n, \%) & $3(30)$ & $5(12.5)$ & 0.33 \\
\hline Hypertension (n, \%) & $4(40)$ & $35(87.5)$ & 0.004 \\
\hline Diabetes (n, \%) & $5(50)$ & $24(60)$ & 0.56 \\
\hline Dyslipidemia (n, \%) & $2(20)$ & $19(47.5)$ & 0.16 \\
\hline Current Smokers (n, \%) & $5(50)$ & $13(32.5)$ & 0.30 \\
\hline Family history of coronary artery disease (n, \%) & $2(20)$ & $2(5)$ & 0.17 \\
\hline
\end{tabular}

TABLE 1: Baseline Characteristics of Cases and Controls among Acute Myocardial Infarction Patients

$\mathrm{SD}=$ Standard Deviation

For linear regression, scale examinations of the continuous variables (age and numeric pain scale) were done which revealed no difference in the value of $\mathrm{R}^{2}$ at the univariate level. Hence, they were kept as a continuous variable for the multivariable linear regression model. A stepwise method was opted for multivariable modeling to build a parsimonious model with maximum $\mathrm{R}^{2}$. The intensity of pain was 2.6 units higher among patients with diagonal branch vessel disease (cases) as compared to those with epicardial vessel disease (controls) [95\% CI: 1.67 - 3.46], adjusted for all other covariates in the model (Table 2). Confounding and interaction were checked for diabetes, hypertension, gender, and age but was not found significant.

\begin{tabular}{|c|c|c|c|}
\hline Variables & Adjusted $\beta$-coefficient & 95\% Confidence Interval & p-value \\
\hline Diagonal branch vessel (cases) & 2.6 & $1.67-3.46$ & $<0.001$ \\
\hline Family history of CAD & 1.2 & $-0.06-2.4$ & 0.06 \\
\hline Age (years) & -0.01 & $-0.04-0.1$ & 0.2 \\
\hline Hypertension & 0.46 & $-0.48-1.4$ & 0.33 \\
\hline Type II Diabetes & -0.2 & $-0.86-0.44$ & 0.52 \\
\hline
\end{tabular}

TABLE 2: Association between Intensity of Pain and Diagonal Branch Vessel Disease among Acute Myocardial Infarction Patients

$\mathrm{CAD}=$ Coronary Artery Disease

Pain perception of patients with a family history of coronary artery disease was slightly higher (adjusted $\beta$ coefficient: 1.2 ), however, the difference was marginally statistically insignificant (p-value: 0.06 ). There was no association of pain intensity with age, hypertension, and diabetes. Future studies may look into a larger sample population from multiple centers to explore the impact of family history and knowledge about the symptoms of AMI among these patients.

\section{Discussion}

This preliminary study highlights a distinct clinical feature of acute myocardial infarction patients with diagonal branch as an infarct-related artery. We observed AMI patients present with severe chest pain without evidence of disease in any major epicardial vessels on the coronary angiogram in our practice. On the contrary, we found disease in one of the branch of major epicardial vessels, which was mostly a diagonal branch causing severe pain. From the review of the existing literature, we know that the severity of chest pain per se does not correlate with the severity of CAD [13]. In addition, patients with isolated branch vessel disease have clinical presentations that are indistinguishable from those of patients with coronary disease 
involving the major vessels $[9,14]$. Evidence regarding the association of pain severity and diagonal branch vessel is very limited and has not been studied clinically. Hence, we decided to test this hypothesis in this pilot study.

Our preliminary data confirms our observation that patients with diagonal branch vessel CAD have more severe chest pain in the condition of AMI. One of the possible pathophysiologies can be explained by the diameter of the vessel. The delivery of oxygen-rich blood to the myocardium is influenced by coronary artery diameter, the presence of collaterals and perfusion pressure determined by the pressure gradient from the aorta to the coronary arteries [15]. Due to a smaller diameter compared to the epicardial arteries, a branch vessel can have persistent vasomotor tone during coronary ischemia, resulting in severe pain due to lack of vasodilators [16]. This phenomenon may also be true for other branch vessel disease however; it was beyond the scope of this study. In addition, the diameter of the vessel has some relation with coronary auto regulatory mechanism during coronary hypo perfusion. Studies in the past have shown that the magnitude of dilatation during coronary hypo perfusion is inversely related to the initial diameter of the vessel [16]. However, it is yet to be known whether the severity of pain is due to the difference in the distribution of pain receptors in different areas of the heart and will serve basis for basic sciences studies in the future [17].

The strength of our study is that it highlights the evidence on the association of pain and diagonal branch vessel disease emphasizing on possible mechanisms between pain pathways and ischemic territories being sensitive to particular branches or vessels. However, the study has certain limitations inherent to the design of retrospective studies and cannot determine causality. As the condition under study is less common (AMI patients with a diagonal branch as culprit vessel), the single-center study contributed to a limited number of cases, while a random selection of controls permitted us to limit selection bias. Future studies involving multiple centers may make this primitive population more accessible for exploration and may be proposed to observe a difference in pain perception between the two groups incorporating knowledge of AMI symptoms and total ischemic time.

\section{Conclusions}

To conclude, our research suggests that if patients present with severe chest pain without significant disease in the major epicardial vessels, one should always look for disease in the diagonal branch vessel, especially in the absence of significant ECG changes. A more aggressive strategy towards the management of chest pain in patients with diagonal branch vessel disease may be required and may lead to higher patient satisfaction and comfort.

\section{Additional Information \\ Disclosures}

Human subjects: Consent was obtained by all participants in this study. Ethics Review Committee, the Aga Khan University issued approval 4358-Car-ERC-16. Ethical approval was sought from Ethics Review Committee, AKU (4358-Car-ERC-16) for this study, which granted exemption for ethical approval and permitted to review hospital records for the study. . Animal subjects: All authors have confirmed that this study did not involve animal subjects or tissue. Conflicts of interest: In compliance with the ICMJE uniform disclosure form, all authors declare the following: Payment/services info: All authors have declared that no financial support was received from any organization for the submitted work. Financial relationships: All authors have declared that they have no financial relationships at present or within the previous three years with any organizations that might have an interest in the submitted work. Other relationships: All authors have declared that there are no other relationships or activities that could appear to have influenced the submitted work.

\section{Acknowledgements}

We would like to acknowledge the support of Health Information Management System's team at the Aga Khan University Hospital for their continued support in the extraction of the data for this study.

\section{References}

1. WHO cardiovascular diseases (CVDs) fact sheet. (2017). Accessed: December 06, 2017: https://www.who.int/news-room/fact-sheets/detail/cardiovascular-diseases-(cvds).

2. Malik MA, Khan SA, Safdar S, Taseer IU: Chest pain as a presenting complaint in patients with acute myocardial infarction (AMI). Pak J Med Sci. 2013, 29:565-568. 10.12669/pjms.292.2921

3. Thygesen K, Alpert JS, Jaffe AS, Simoons ML, Chaitman BR, White HD: Third universal definition of myocardial infarction. Circulation. 2012, 126:2020-2035. 10.1161/CIR.0b013e31826e1058

4. Hofkamp SE, Henrikson CA, Wegener ST: An interactive model of pain and myocardial ischemia. Psychosom Med. 2007, 69:632-639. 10.1097/PSY.0b013e318151a4bd

5. Krantz DS, Hedges SM, Gabbay FH, et al.: Triggers of angina and ST-segment depression in ambulatory patients with coronary artery disease: evidence for an uncoupling of angina and ischemia. Am Heart J. 1994, 128:703-712. 10.1016/0002-8703(94)90268-2

6. Foreman RD, Garrett KM, Blair RW: Mechanisms of cardiac pain. Compr Physiol. 2015, 5:929-960. 10.1002/cphy.c140032 
7. Wells N, Pasero C, McCaffery M: Patient Safety and Quality: An Evidence-based Handbook for Nurses . Hughes R (ed): Agency for Healthcare Research and Quality, Rockville; 2008.

8. Wells N, Pasero C, McCaffery M: Improving the quality of care through pain assessment and management . Patient Safety and Quality: An Evidence-Based Handbook for Nurses. Hughes RG (ed): Agency for Healthcare Research and Quality, Rockville; 2008. 37.

9. Lujinović A, Ovčina F, Voljevica A, Hasanović A: Branching of main trunk of left coronary artery and importance of her diagonal branch in cases of coronary insuficiency. BJBMS. 2005, 5:69-73. 10.17305/bjbms.2005.3274

10. Merz CNB, Shaw LJ, Reis SE, et al.: Insights from the NHLBI-sponsored women's ischemia syndrome evaluation (WISE) study: part II: gender differences in presentation, diagnosis, and outcome with regard to gender-based pathophysiology of atherosclerosis and macrovascular and microvascular coronary disease. J Am Coll Cardiol. 2006, 47:S21-S29.

11. Kohn MA, Kwan E, Gupta M, Tabas JA: Prevalence of acute myocardial infarction and other serious diagnoses in patients presenting to an urban emergency department with chest pain. J Emerg Med. 2005, 29:383-390. 10.1016/j.jemermed.2005.04.010

12. Khan MS, Jafary FH, Faruqui AM, Rasool SI, Hatcher J, Chaturvedi N, Jafar TH: High prevalence of lack of knowledge of symptoms of acute myocardial infarction in Pakistan and its contribution to delayed presentationto the hospital. BMC Public Health. 2007, 7:284-292.

13. Wellens HJJ, Gorgels APM, Doevendans PA: Determining the size of the area at risk, the severity of ischemia, and identifying the site of occlusion in the culprit coronary artery. The ECG in Acute Myocardial Infarction and Unstable Angina. Wellens HJJ, Gorgels APM, Doevendans PA (ed): Springer, Boston; 2002. 245:5-42. 10.1007/0-306-48202-9_2

14. Iwasaki K, Kusachi S, Kita T, Taniguchi G: Prediction of isolated first diagonal branch occlusion by 12-lead electrocardiography: ST segment shift in leads I and aVL. J Am Coll Cardiol. 1994, 23:1557-1561. 10.1016/0735-1097(94)90656-4

15. Malyar NM, Gössl M, Beighley PE, Ritman EL: Relationship between arterial diameter and perfused tissue volume in myocardial microcirculation: a micro-CT-based analysis. Am J Physiol Heart Circ Physiol. 2004, 286:H2386-H2392. 10.1152/ajpheart.00682.2003

16. Sun H, Mohri M, Shimokawa H, Usui M, Urakami L, Takeshita A: Coronary microvascular spasm causes myocardial ischemia in patients with vasospastic angina. J Am Coll Cardiol. 2002, 39:847-851. 10.1016/S0735-1097(02)01690-X

17. Leach A, Fisher M: Myocardial ischaemia and cardiac pain-a mysterious relationship. Br J Pain. 2013, 7:2330. $10.1177 / 2049463712474648$ 